\title{
Supporting transition to parenthood in Aotearoa New Zealand
}

Irene de Haan University of Auckland, New Zealand

\begin{abstract}
INTRODUCTION: Recent rhetoric about investing in 'vulnerable' children disregards the reality that the magnitude of change accompanying adaptation to parenthood makes all infants and their families vulnerable. This article reports the findings of a small-scale qualitative study of Aotearoa New Zealand parents' experiences of transition to parenthood and their views on support received or wished for.
\end{abstract}

AIM: To gain insight into how adaptation to life with a baby is experienced and how support could be improved.

METHODS: The study involved sequential in-depth interviews with each of 25 socioeconomically diverse first-time mothers and a single set of interviews with 11 fathers and one grandmother. Narrative analysis was used to discern themes.

FINDINGS: Participants expressed surprise regarding challenges inherent in adaptation to parenthood. While they deeply appreciated support from Lead Maternity Carers, they saw later professional support as "for the baby". They found little support to deal with problems experienced, which encompassed financial, accommodation, mental health and relationship issues.

CONCLUSION: A true investment approach would build on families' commitment to making beneficial change for the sake of their baby. Well-being could be promoted by longer stays in maternity facilities and straightforward information and coaching about dealing with change as well as about infant care. Fathers' needs should be carefully considered to help secure engagement in life with a baby. There is a key role for family support social work in designing relevant services and in meeting a need for 'someone to talk to' for early help to process change and resolve problems.

KEYWORDS: first-time parents; family support; narrative methods

AOTEAROA

NEW ZEALAND SOCIAL WORK 28(3), 4-14.

CORRESPONDENCE TO: Irene de Haan

i.dehaan@auckland.ac.nz

\section{Introduction}

Adaptation to parenthood brings changes in lifestyle, roles, responsibilities and relationships (Seah \& Morawska, 2016; Wilkins, 2006). Life is suddenly dominated by the baby's needs (Ahlborg \& Strandmark, 2001). Breastfeeding is often problematic (Ingram, Johnson, \& Greenwood, 2002).

Broken sleep and anxiety about infant health or development are usual. Inability to soothe a fretful infant produces an unfamiliar type of helplessness. Cowan, Cowan \& Schultz (1996, p.30), taken aback by high levels of stress incidentally reported by new parents in couples groups, say "It would be tragic if the real difficulties families face as they attempt to raise young children were to be ignored on the assumption that help is needed only by those in more traditionally high-risk samples". 
Many new parents need support to manage difficulties, whether transitory or persistent. While life with a baby can be delightful, it is a challenge, sometimes complicated by stress or illness. Around 20\% of mothers and 5\% of fathers experience perinatal depression or anxiety (Epifanio, Vitalba, De Luca \& La Grutta, 2015). Poverty, addiction and inadequate housing all make adaptation to parenthood harder. Conversely, the demands of caring for a baby may undermine capacity to deal with chronic problems. This article draws on qualitative research completed in 2010 (de Haan, 2011) exploring the experiences and views of firsttime parents in Aotearoa New Zealand.

Aotearoa New Zealand has persistently high rates of family violence and child maltreatment. Victims are repeatedly let down by the system (The Office of the Children's Commissioner, 2016; Wilson, Smith, Tolmie \& de Haan, 2015). Abuse within families causes harm that is cumulative and long-lasting (Broadley, 2014; Munro, 2011). If social work is to enact its commitment to social justice we must devise strategies to promote well-being and prevent harm. It seems sensible to offer support as families embark on raising children, but understanding how best to do so requires understanding of the perspective of parents themselves.

Aotearoa New Zealand has an established system of free maternal and infant health services. Women are encouraged to choose a Lead Maternity Carer (LMC) to provide consistent antenatal and perinatal care. Most LMCs are community-based midwives. After a few postnatal home visits LMCs hand over care to 'well child' nurses, offering most mothers home visits for very young infants, then drop-in clinics. Traditionally these nurses worked under the banner of the venerable New Zealand Plunket Society, established in 1907 to improve infant health. Other agencies now provide similar services, notably Māori providers. However, all services must conduct 4-6 week child development assessments, recently found to be insensitive to Māori and
Pacific people and to disregard "fundamental differences in parenting approaches between indigenous and other population groups" (Litmus, 2013, p.171).

Munro's review of child protection in England (2011) identifies a pressing need for early intervention, using a partnership approach to resolve problems. Aotearoa New Zealand families would have had more chance of finding such help in the 1980s and 1990s, when non-profit agencies were contracted to support families under stress, a term covering a multiplicity of circumstances including financial strain, mental illness, relationship problems and past trauma (Munford \& Sanders, 1999). These services were intended to be easily accessible and responsive to self-identified needs. The rationale for this accessibility was that overtly recognising that all families may struggle at times reduced shame and stigma, making it easier for families to seek help. Family-centred practice of this kind reflected international interest in supporting families to tackle problems that undermine ability to provide a secure environment for children. In Aotearoa New Zealand the focus has narrowed to target, as in other countries, families considered troublesome and troubled and 'high risk and high cost' (Churchill \& Sen, 2016).

Cost concerns are implicit in the recently completed review of Aotearoa New Zealand's statutory child protection agency, Child, Youth and Family. The Modernising Child, Youth and Family Panel (2016, p. 38) uses an actuarial model to estimate costs of poor life outcomes (e.g. incarceration; income support receipt) expected to eventuate from childhood vulnerability, a term currently ubiquitous in Aotearoa New Zealand policy rhetoric. Since it is the family environment that is envisaged as undermining children's well-being, it is odd that no coherent strategy is proposed for addressing problems that beset families and distract them from the myriad tasks and interactions involved in raising children. What this child-focused investment approach will mean in practice is unclear. 
What is clear, however, is that when families perceive services as intrusive or judgemental they are likely to be wary of telling professionals their whole story. As Morris (2013, p.203) says, they "may hold back in revealing their needs, fearful of the ensuing interventions". Yet knowing the whole story enables professionals to provide the reliable, responsive support that underpins constructive change (Morris, 2013).

Support is categorised as informal, semiformal and formal. Personal networks provide informal support including comfort, advice and practical help. Semi-formal support includes skilled peer support and member-run groups, sometimes facilitated by service providers. Formal support is service provision requiring professional expertise. Introducing the UK 'Supporting Parents' studies, Quinton (2004, p.22) concludes: "Support is complex to assess, to get right and to deliver", particularly in view of "differences in individual and cultural ideas of what satisfactory parenting is, how and when this needs support and who should decide that".

Contemplating the importance of understanding the context of parents' lives, Duncan, Bowden and Smith (2005, p.14) reject "a top down expert to parent approach", recommending partnership models instead. From a social work perspective, partnership is inherent in strengths-based family support models (Scott \& O'Neill, 2003) and in the respectful relationship-based practice promoted by Featherstone, White and Morris (2014). Yet "remarkably little is known about how families reflect on their lives and experiences and the implications... for social work practice" (Morris, 2013, p.199). Professionals, policy analysts and service providers may be blinkered by assumptions embedded in their own culture and background and so misconstrue the realities of parents' lives and attempt to impose inappropriate concepts or practices (Silberberg, 2001; Waller, 2001). Reporting research about Māori sudden infant death, de Joux (1998. p.2) explains that antenatal services available to Māori women did not meet their spiritual or cultural needs and expectations:

Many of the Māori women interviewed were adamant that traditional practices and customs should be reintroduced and incorporated in any programmes that were developed... The concept of educating the woman only and maybe the partner is not realistic when in the whanau environment she may be one of several members of the whanau that are responsible for the care and support of the baby.

If services are designed and delivered according to assumptions about recipients' needs and preferences, they will suit only a relatively narrow band of potential users, who are probably, in terms of background and circumstances, not unlike the designers of the support: mature, educated, affluent, culturally mainstream. Pondering why young women viewed antenatal classes as "not for them", Cliff and Deery (1997, p.144), go so far as to wonder "whether older married mothers are actually the preferred clientele of professionals responsible for designing and delivering these services". Clearly there is potential for misalignment between the nature and style of services and the needs and preferences of people expected to use those services. This suggests a need for input by potential users at the design stage.

My study explored how new parents experienced adaptation to parenthood. Participants were encouraged to tell their stories in their own way but prompted to talk about support, including informal support to provide insight into how helpful aspects of informal support might improve formal services. Ideas for change or innovation were welcomed. The research questions were:

- What was the experience of first time parents in a range of circumstances in Aotearoa New Zealand today as they adapted to life with a baby? 
- What factors did a diverse set of first time parents experience as helpful or unhelpful as they dealt with challenges encountered during transition to parenthood?

- What are the implications for policy and service delivery?

\section{Methods}

Transition to parenthood is deeply personal so a narrative methodology was appropriate for this qualitative study. People commonly tell stories to communicate personal experience (Maines, 1993; Sandelowski, 1991) and women commonly want to talk about childbirth (Olin \&Faxelid, 2003). A theoretical base for the study is the ecological perspective central to Patterson's (2002) conceptualisation of family resilience as related both to family processes and to risks and opportunities in social systems.

In Aotearoa New Zealand, as elsewhere, the age range of first-time mothers spans the teenage years to women over 40 . To reflect this, the purposive sample comprised 27 first-time mothers aged 16-40, resident in two demographically different areas of Auckland city as well as in a semi-rural area within 90 kilometres of Auckland and a rural area about 200 kilometres from Auckland. Recognising that transition to parenthood is a process over time the study involved three sequential indepth interviews with each woman, the first in late pregnancy; the second three months after the birth; the third around the baby's first birthday. To make interviews convenient and comfortable, participants were invited to choose the location, usually their home.

Participants were initially recruited through childbirth educators. I was invited to briefly visit classes to describe the study and leave information sheets. I then contacted women who registered interest. Although this strategy was successful, women thus recruited were mostly over 26 : few younger women attended classes. To recruit young participants, and also in rural areas lacking childbirth education, I invited midwives to display flyers about the project and give information sheets to interested women. An intermediary consent form allowed them to pass women's contact details on to me. This strategy attracted participants from different areas and in various circumstances. However, only four were Māori and two of Pacific Island heritage; most were Pakeha (New Zealand European) or European migrants. One participant dropped out of the study before the second interview and another was unavailable, having moved into a women's refuge to escape an abusive partner. Two younger women were untraceable for the third interview. The experience of significant others was included, both through women's reports and through a set of single interviews, mid-way through the project, with 11 partners, all men, whom participants invited to participate. The mother of one of these men was also interviewed. Ethical approval was obtained from Massey University Human Ethics Committee and the Northern Regional Health Ethics Committee. In case interviews raised potentially harmful issues, I carried details of sources of help. Confidentiality was paramount. I undertook all the interviews and transcription. Pseudonyms (followed by age) distinguish participants from one another.

An interview schedule was used flexibly, enabling participants to discuss whatever was important to them. Analysis was done by parsing narratives (Gee, 1991) through repeated listening to participants' taped narratives to hear how each speaker introduced and concluded stories by using "entrance and exit talk" (Riessman, 1993, p.58) and used pauses and shifts in tone to build lines (units of meaning) into stanzas (groups of lines with similar content), constructing stories woven into narratives. The stories contain much everyday detail. Themes were discerned through coding and categorizing lines within stories.

\section{Limitations}

A limitation of the study is its small scale. Only six of 27 participants recruited were non-European. Experiences and views 
presented can only be indicative of those of first-time parents in Aotearoa New Zealand. Voluntary participation may have favoured recruitment of people coping well rather than struggling to cope. However, several participants encountered extremely challenging circumstances at various times. Recognizing its limitations, the study's aim was "informative" research (Schoenberg, Shenk, \& Kart, 2007, p.11) in that studies employing "a limited number of cases with rich contextual data and no pretensions of generalizability may be ... informative for the specific research question being addressed".

\section{Findings}

Key themes are presented under the following conceptual categories: A lifechanging experience; learning coping strategies; adapting to fatherhood; balancing parenting, employment, isolation.

\section{A life-changing experience}

Participants described transition to parenthood as life-changing. Celia (37) commented: "Our main focus now is [Baby]. Most of the stuff we do or eat or go to is around her". Tom (37) highlighted relationship change: "The husband is no longer the centre of the wife's attention, it's as simple as that, no longer the be all and end all, the baby is". Since many stories recount difficulties it is important to note that generally participants found caring for their babies enjoyable and were determined to be "good" parents. This sentiment applied across the age range but was most poignantly expressed by participants for whom childhood was hard. The stories of Abby (19) and Kim (18), both maltreated as children, suggest that becoming a parent can be a turning point (Rutter, 1987), reorienting a life trajectory towards well-being. Echoing findings of a study of adolescent Latino parents (Dornig et al., 2009), Abby said: "Your life changes dramatically. You can't have the same friends you had doing drugs; you've got to be a role model for your little one. It's a 24/7 job".
However, Dawn (17), also keen to "be a good mum", developed postnatal depression, left her partner Kent (18), and was "staying around everywhere, in motels and stuff", seeing the baby only intermittently. Kent's mother lamented the lack of professional support available to Dawn. Kent thought schools should teach about parenthood: "It's hard, coming from a freedom life to having a baby. You're responsible. Some people think it's quite easy. It's not".

Many found the shift from "a freedom life" hard. Beforehand they could hardly see past the birth: most embarked on parenthood with scant knowledge of what parenthood entails. As Cara (31) said: "Suddenly you've got this baby and you think, well, I haven't actually read anything about what to do now".

\section{Learning coping strategies}

Participants typically were taken aback by the "constantness" of infant care. Jess(29) explained:

I don't think you can actually appreciate until you've got one, the constantness of it. We're in the position where we don't have family around, it makes it harder, we don't get a break. But I really enjoy looking after him. The day goes so quickly.

"Floundering in the deep end" metaphors were used by several participants to express exhaustion, confusion or panic. Amy (19) benefitted from informal and formal support, including phone help lines like Plunketline.

When he's sick I just stress. I've got no clue. I panic. I ring Mum. I ring my friend she's got two kids, she knows basically everything. She gives a couple of ideas and I try them. I ring Plunketline but sometimes you have to wait a bit. [Baby] fell off his changing table even though he was strapped in. I felt worse when I went to the doctor's: 'What will they think? I dropped my baby!' He was fine. And they were nice. 
Some participants expected to learn infant care techniques while recovering from giving birth and were astonished, even outraged, when this did not happen. Some were more fortunate. Kim (17) hated being in hospital, where she felt ignored and "enclosed" and against her midwife's advice left too soon after a caesarean:

The next day I rang [midwife] and asked if I could go to the birthing unit. I got there, which was really good because I had all the support I needed. I thought I'd have to go in three days but she said I could stay until I'm ready to leave. [Partner] was allowed to stay. It was really good.

Participants generally praised birthing centres, which provide facilities for LMCs to manage relatively straightforward births, with women staying around three days. Participants felt safe with their "own midwife", by then regarded as a trusted advisor. They appreciated the respectful, upbeat atmosphere and the willingness of staff to welcome partners to stay overnight. Some men were taught basic infant care and women reported receiving patient, kindly help with establishing breastfeeding.

What participants most wanted from personal networks was practical support. Food provided by friends and family was deeply appreciated, as was help with shopping. However, some family members were unavailable due to employment commitments, living miles away, or estrangement. Participants craved appraisal support (Warren, 2005), that is, someone they trusted noticing their developing competence, but this was rarely forthcoming. Interestingly, younger participants seemed to more easily adapt to life with a baby than older participants, who typically were anxious about "doing it right" (Wilkins, 2006). While LMC visits were helpful, LMCs were often elsewhere when support was actually needed. Participants were ambivalent about well child services, seeing nurses as "there for the baby" with no role in supporting them generally. It was reassuring to know the baby's development was normal, but not always easy to get reassurance. Amy (19) said: "I've tried going there to get him weighed but either they're closed or they're busy". Several described encounters with "bossy" or "directive" nurses. Fay (34) told a story about avoiding "problems" by pretending to do as the nurse suggested. Ange (33) never returned to a clinic after the nurse rejected her explanation of why her tired baby did not demonstrate a developmental milestone.

Semi-formal support was of limited use. Childbirth educators and well child services in Aotearoa New Zealand encourage peer support by establishing neighbourhood coffee groups for mothers, who then organize groups themselves. Only two participants enjoyed their coffee groups. Ange (33), experienced hers as "a forced situation" where she found no-one compatible. Nor were groups always accessible. Julie (23) explains:

The antenatal class have a coffee group but its miles away, I just thought there's no point. There are probably things out there but I kind of slipped through... Occasionally I spend the day without seeing Mum or anyone and I'm kind of 'This isn't very fun'.

A problem with building semi-formal support on childbirth education is that this best suits older, more affluent women. Young participants felt uncomfortable in classes and had no incentive to stay connected. This corresponds to a study indicating that women who attend childbirth education want to "meet people like me" while non-attenders think classes are "not for people like me" and fear being "looked down upon" (Cliff \& Deery, 1997, p.143). Two teenage participants enjoyed childbirth education designed for their age group, but spaces there were limited. 
Several participants said that "teamwork" helped with adapting to life with a baby. Jan(33) explained how she and her partner coped with their baby's colic: "It's working together rather than [having] defined roles. It's been a life-changing experience but we've adapted. We've been a team since we got married, now we've got a new member". However, services apparently only nominally recognised men as "hands-on dads".

\section{Adapting to fatherhood}

Lack of support for men was a common complaint. Men often felt ignored or undervalued, "spare" in hospital delivery rooms and unwelcome afterwards. Matt (38) believed men should be warned they would get little attention: "The guy's invisible... they've got a little area for dads to make sandwiches and stuff [but] the nurses and doctors are...not so much rude but they won't even acknowledge you're talking". Telling a story about a childbirth educator encouraging men to "talk about men's stuff", Roz (23) said this failed because the educator was female: "It would be better to have a bloke for the blokes". Several men were disappointed to find themselves "redundant" at first, especially with breastfed babies. Matt(38) explained:

During the whole pregnancy there's the two of you, these messages, you're in it together. Then the baby comes - and it's the two of them, mum and baby, and you just kind of, well, make dinner, wash dishes...Guys take a couple of weeks feeling anxious, absolutely helpless, before the crying doesn't get on your nerves. I think a really good message for dads is, it actually gets much better after, like, four months.

Some men worried about replicating parenting demonstrated by their own fathers. One attended private counseling, but others worried silently without help to process experiences of destructive behaviour patterns.

\section{Balancing parenting, employment, isolation}

As the year progressed women felt increasing pressure to return to work, several agonizing over relative advantages of earning money, regaining status and being a "full-time mum". Some professional women felt "left behind" or that they were "becoming a bit dim", as Jess (29) said. Some fathers were still working long hours. Crises caused strain, as Abby(19) recounted:

Mostly we just manage to get by. There's been the odd time when the cupboard was quite bare, but we've fed [Baby], used the money for her [when] bills got on top of us. But bills are important, like the roof over our heads, the power. [Partner] took a week unpaid leave when we were in hospital. So we were a bit down on money...We got a bit grumpy, hadn't had the nutrition. We'd go over to his sister's for dinner.

Many participants were "just getting by". Working part-time meant "prioritising" or "cutting back". Those on low incomes or Benefits found "every $\$ 5$ counts". Those with high incomes usually had high outgoings. Returning to employment caused angst. Clare (31), who felt "nobody cares what I do with my day", explained her dilemma:

Going from a working woman to a mum, feeling bored and lonely, felt like a loss. I was surprised how difficult that was... The company has been flexible, brilliant but I think my manager's found it difficult. I suspect that often happens. I don't feel I can complain but it hasn't been a total success. I'm actually at the stage I'd rather be at home. I'm working four days - four days the manager can think of you as a real person, three days and I'm a 'part-time mum'.

Dee (20), a waitress, also found her manager's attitude subverted an overtly supportive arrangement. 
Others felt "bored and lonely" too. Zoe (17), renting a house in a poorly resourced suburb, was sure there were young mothers around as lonely as herself and "needing to get out of the house". Having heard that a local agency was planning a group for "first-time mums that don't know what to do, practical stuff", she was upset this never eventuated. Some participants made friends through childbirth education, enjoying outings, emotional support and reciprocal babysitting. Services found useful were drop-in Plunket Family Centres, offering company and guidance but available in only a few areas; and Mainly Music, a churchbased activity evidently widely available and used by a range of people with toddlers. Mostly, however, women told stories of isolation and diminished status.

\section{Discussion}

The study suggests that policy rhetoric about valuing children's well-being is currently not matched by commitment to supporting parents. Otherwise, as Cheryl (26) noted, a "Parent Subsidy" equivalent to Childcare Subsidy would recognise that parenting is valuable. Cheryl thought it absurd that as a "full-time mum" she was deemed "not working", yet, if she had a job, childcare workers would be paid to look after that same child. Participants commonly struggled financially. Paid parental leave was a boon for those eligible but ineligibility caused indignation, especially when women were employed just slightly less than the year required. Some fathers had little time off work around the birth; one in effect had none. Yet no-one suggested paid paternal leave, perhaps because this might mean less maternal leave.

An ecological view (Patterson, 2002) of support for adaptation to parenthood indicates that despite free, universal maternal and infant health care, the current Aotearoa New Zealand system lets families down. In particular, LMC support stops so abruptly that women may feel abandoned, even shocked. From this point onwards service provision is experienced as "for the baby". As Julie (23) realised: "We were just another family - it's on to the next one now".

At societal level there is apparently attitudinal disjunction. Policy rhetoric, transmitted through the media, directs public sympathy towards vulnerable children and positions the child protection system as poised to swoop in and castigate parents deemed failing. Meanwhile, participants plainly felt that raising children is commonly regarded as a mundane endeavour, with efforts to do well hardly noticed, far less applauded. While the notion 'It takes a village to raise a child' is often espoused, responsibility actually rests with parents. As Cheryl (26) mused, parents are expected to "just cope" and this makes it hard to admit to needing help; struggling to cope is internalised as "failing to do something anyone can do". An individualistic worldview may occasionally hinder help-seeking: Julie (23) said:

"[Partner] doesn't like help from people, he thinks when he's achieved something on his own, he's achieved it". This suggests that policy makers and service providers must not only be aware that pride or shame may deter help-seeking, but also be vigilant against the possibility of exacerbating shame. Participants' narratives indicate that when new parents experience service provision as disrespectful or insensitive they simply avoid providers or fake compliance. This indicates a worrying lack of the trust that enables people to tell their whole stories. If we are to do better in stopping family violence and child maltreatment in Aotearoa New Zealand we must offer services that families trust, so problems do not remain hidden. We must also ensure that intervention is sophisticated enough to respond to the complexity and diversity of families' lives.

The study suggests that respectful, responsive interaction is vital in enabling health professionals to fulfil their important role in facilitating transition to parenthood. Enabling women to stay longer in maternity 
facilities could strengthen the turning point potential of becoming a parent. Possible strategies include reinforcing maternity facilities' welcoming, congratulatory atmosphere; providing straightforward information about common problems; and spending more time coaching new mothers and fathers in infant care. Using current rhetoric, this would be an investment. Participants' stories repeatedly recount episodes where men felt "ignored", "invisible", "useless" or "helpless", resulting in considerable disappointment and chagrin. Potentially this could tarnish the experience of becoming a father and compromise the task-sharing notion of teamwork. Feeling powerless may just possibly be a factor in infant maltreatment. This proposition warrants further study.

There was notable change in multiple aspects of participants' lives. Problems encountered extended beyond infant care into financial strain, housing difficulties, relationship problems, physical and mental illness and effects of past trauma. Many were unaware of tax credits and other financial assistance available, or unsure how to apply. Many benefited from informal support in the form of practical help; material assistance such as nappies or food; or down-to-earth emotional support, the latter commonly delivered in loving, no-nonsense ways, making them laugh and defusing anxiety. For example, when Tania (20) worried about her baby blowing bubbles her sister said: “It's rabies!... I'm joking, all babies blow bubbles".

An intriguing implication of the study is the usefulness of social work expertise in developing services to make the most of prospective parents' determination to be good parents. As Quinton found (2004, p.189) participants preferred services that "took their views and needs seriously, listened to them and were emotionally supportive as well as practically helpful". Services to meet participants' expressed needs would facilitate access to material support; provide reliable advice, perhaps through events offering company; cater for both men and women and support the notion of teamwork; offer skilled emotional support; and, importantly, be delivered in a relaxed, respectful style, perhaps at neighbourhood family centres. More specifically, across the entire age range participants wanted "someone to talk to", an objective, supportive listener who could understand them, comprehend their concerns, offer information and guidance presented as options rather than directives, and, as Tania (20) said "get the necessary help, have the contacts -almost like, not a counsellor, someone just to talk to. Even a couple of times would be enough for them to see a problem". Karen (40) explained "You want to talk when you've just given birth, you really do, but nobody else really wants to". Pondering what her son's partner needed when first experiencing postnatal depression, Lisa said:

She needed someone that could come round. She found it very upsetting when her midwife stopped, after the six weeks. You get so attached then all of a sudden it's like 'You're in Plunket's care now'. For the baby it's good, but Plunket seems more about Baby than Mum. That's what needs changed. It's like a feeling of abandonment for some mums. I reckon we need people that train in that field of work, to see if you've got worries - if no worries that's brilliant, but just to know they're like a friend coming for a chat but like a therapist. And if something's wrong they might be able to help and guide them.

This suggests a need for the responsive, relationship-based practice described by Featherstone et al. (2014) and Morris (2013). If introduced by LMCs or other trusted people, and available to all new mothers, home visits by family support social workers could provide new parents with early intervention. (Munro, 2011) in a comfortable, non-stigmatizing way.

In considering support for new parents, Ungar's (2008) concept of navigation and 
negotiation is useful. Navigation refers to capacity to seek support and availability of support sought. Negotiation denotes adjustment of style, delivery and purpose of services to suit background and culture. Although participants wanted to navigate to, or access support they were thwarted by limited availability or absence of services. The crux of negotiation is designing services to meet potential users' actual needs. Apart from limited childbirth education for teenagers there was little evidence of negotiation having taken place. No-one mentioned services designed to meet cultural needs. Yet, if services are misaligned with users' background or culture then professionals may mistake normative behaviours for risk factors (Waller, 2001) undermining rather than reinforcing confidence and competence and potentially causing rather than assuaging problems.

\section{Conclusion}

Recently the term vulnerable has been widely used to designate children and families at risk because of their circumstances. Reclaiming a less stigmatising use of vulnerable positions all infants as vulnerable, being utterly dependent on adults for nurturing and safety, and all new parents as vulnerable, facing new responsibilities and profound change. Every family's circumstances are different. Transition to parenthood is idiosyncratic as well as complex.

Social work is well-placed to understand and respond to complexity (Duvnjak \& Fraser, 2013). The social work knowledge base is applicable to supporting families in challenging and complicated circumstances; social workers' practice wisdom can constructively contribute to developing services designed to provide early help to stop difficulties becoming entrenched. So that services suit actual needs parents should be involved in the design of these services. However, services likely to appeal to parents would recognise that they want to do their best for their babies, provide a mix of information, practical and emotional support and involve fathers. By building on existing universal services a true investment could be made in the well-being of infants and their families. Childbirth education and maternity care could be used to facilitate more learning about looking after an infant and about changes inherent in adaptation to parenthood. Softening the loss of a woman's close relationship with her LMC could be achieved by offering a visit or two by a family support social worker. Further research could usefully investigate any links between fathers' sense of helplessness and infant maltreatment.

\section{References}

Ahlborg, T., \& Strandmark, M. (2001). The baby was the focus of attention: First-time parents' experiences of their intimate relationship. Scandinavian Journal of Caring Sciences, 15(4), 318-325. doi: 10.1046/j.14716712.2001.00035.x

Broadley, K. (2014). Equipping child protection practitioners to intervene to protect children from cumulative harm: Legislation and policy in Victoria, Australia. Australian Journal of Social Issues, 49(3), 265-284.

Churchill, H., \& Sen, R. (2016). Introduction: Intensive family support services: Politics, policy and practice across contexts. Social Policy \& Society, 15(2), 251-261. doi: $10.1017 / \mathrm{S} 1474746416000026$.

Cliff, D., \& Deery, R. (1997). Too much like school: Social class, age, marital status and attendance/nonattendance at antenatal classes. Midwifery, 13(3), 139-145. doi: 10.1016/S0266-6138(97)90004-8

Cowan, P., Cowan, C., \& Schultz, M. (1996). Thinking about risk and resilience in families. In M. Hetherington, \& E.A. Blechman (Eds.), Stress, Coping and Resiliency in Children and Families. (pp.1-38). Malwah: Lawrence Erlbaum Associates Inc.

de Haan, I. (2011). A Good Start: Supporting families with a first baby. Unpublished PhD thesis, Massey University, Auckland.

De Joux, R. (1998). Matuaranga Haputanga- Antenatal Services for Māori Women. Auckland: Whakawhetu, National SIDS Prevention for Māori, University of Auckland.

Dornig K., Koniak-Griffin, D., Lesser, J., Gonzalez-Figuera, E., Luna, M., Anderson, N., \& Corea-London, B. (2009). "You gotta start thinking like a parent": Hopes, dreams, and concerns of ethnic minority adolescent parents. Families in Society: The Journal of Contemporary Social Services, 90 (1), 51-59. doi: 10.1606/1044-3894.3845

Duvnjak, A., \& Fraser, H. (2013). Targeting the 'hard to reach': Re/producing stigma? Critical and Radical Social Work, 1(2), 167-182. doi: http://dx.doi.org.ezproxy. auckland.ac.nz/10.1332/204986013X673245

Duncan, J., Bowden, C., \& Smith, A. (2005). Early childhood centres and family resilience. Wellington, NZ: Centre for Social Research and Evaluation, Ministry of Social Development. 
Epifanio, M., Vitalba, G., De Luca, C., \& La Grutta, S.(2015). Paternal and maternal transition to parenthood: The risk of postpartum depression and parenting stress. Pediatric Reports, 7(2) 38-44. doi: 10.4081/pr.2015.5872

Featherstone, B., White, S., \& Morris, K. (2014). A marriage made in hell: Early intervention meets child protection. British Journal of Social Work, 44(7), 1735-1749.

Gee, J (1991). A linguistic approach to narrative. Journal of Narrative and Life History, 1(1), 15-19.

Ingram, J., Johnson, D., \& Greenwood, R. (2002).

Breastfeeding in Bristol: Teaching good positioning, and support from fathers and families. Midwifery, 18, 87-101. doi: 10.1054/midw.2002.0308

Litmus (2013). Well Child Tamariki Ora Quality Reviews. Wellington, NZ: Ministry of Health Manatū Hauora: Wellington.

Maines, D. (1993). Narrative's moment and sociology's phenomena: Toward a narrative sociology. The Sociological Quarterly, 34(1), 17-38. doi: 10.1111/j.15338525.1993.tb00128.x.

Morris, K. (2013). Troubled families: Vulnerable families' experiences of multiple service use. Child \& Family Social Work, 18(2), 198-206. doi: 10.1111/j.13652206.2011.00822.x

Munford, R., \& Sanders, J. (1999). Supporting families. Palmerstone North, NZ: Dunmore Press.

Munro, E, (2011). The Munro review of child protection final report: A child- centred system. Final Report: A child-centred system. London: Department for Education.

Office of the Children's Commissioner (2016). State of care, 2016. Wellington, NZ: OCC.

Olin, M., \& Faxelid, E. (2003). Parents' needs to talk about their experiences of childbirth. Scandinavian Journal of Caring Science, 17, 153-159.

Patterson, J.M. (2002). Understanding family resilience. Journal of Clinical Psychology, 58(3), 233-246.

Quinton, D. (2004). Supporting parents: Messages from research. London: Jessica Kingsley Publishers.

Riessman, C. (1993). Narrative analysis. Newbury Park, CA: Sage Publications.

Rutter, M. (1987). Psychosocial resilience and protective mechanisms. American Journal of Orthopsychiatry, 57(3), 316-331. doi: 10.1111/j.1939-0025.1987.tb03541.x.

Sandelowski, M. (1991). Telling stories: Narrative approaches in qualitative research. Journal of Nursing Scholarship, 23(3), 161-166.

Schoenberg, N., Shenk, D., \& Kart, C. (2007). Food for thought: Nourishing the publication of qualitative research. The Journal of Applied Gerontology, 26(1), 4-16.

Scott, D., \& O'Neil (2003). Beyond child rescue. Bendigo, VIC: Solutions Press.

Seah, C., \& Morawska, A. (2016). When Mum is stressed, is Dad just as stressed? Predictors of paternal distress in the first six months of having a baby. Infant Mental Health Journal, 7(1), 45-55. doi: 10.1002/imhj.21546.

Silberberg, S. (2001). Searching for family resilience. Family Matters, 58, 52-57.

The Modernising Child, Youth and Family Panel (2016). Expert Panel Final Report: Investing in New Zealand's Children and their Families. Wellington, NZ.
Ungar (2008). Resilience across cultures. British Journal of Social Work, 38, 218 - 235. doi: 10.1093/bjsw/bcl343.

Waller, M. (2001). Resilience in ecosystemic context: Evolution of the concept. American Journal of Orthopsychiatry, 71(3), 290-297. doi: 10.1037/00029432.71.3.290.

Warren, P. (2005). First-time mothers: Social support and confidence in infant care. Journal of Advanced Nursing, 50(5), 479-488.

Wilkins, C. (2006). A qualitative study exploring the support needs of first-time mothers on their journey towards intuitive parenting. Midwifery, 22, 169-180.

Wilson, D., Smith, R., Tolmie, J., \& de Haan, I. (2015). Becoming better helpers: Rethinking language to move beyond simplistic responses to women experiencing intimate partner violence. Policy Quarterly, 11(1), 25-31. 